Annalisa Baldi · Bruno Franchi · Maria Carla Tesi

\title{
Hypoellipticity, fundamental solution and Liouville type theorem for matrix-valued differential operators on Carnot groups
}

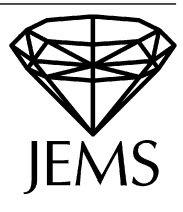

Received May 16, 2007 and in revised form September 26, 2007

\begin{abstract}
Let $\mathcal{L}$ be a non-negative self-adjoint $N \times N$ matrix-valued operator of order $a \leq Q$ on a Carnot group $\mathbb{G}$. Here $Q$ is the homogeneous dimension of $\mathbb{G}$. The aim of this paper is to investigate the relationship between hypoellipticity and maximal hypoellipticity (i.e. sharp $L^{2}$ estimates in appropriate Sobolev spaces), $L^{p}$-maximal hypoellipticity (i.e. sharp $L^{p}$ estimates in appropriate Sobolev spaces for $1<p<\infty$ ), and what we call maximal subellipticity of $\mathcal{L}$ (which is basically a sharp higher order energy estimate).
\end{abstract}

Keywords. Hypoelliptic operators, maximal hypoelliptic operators, subelliptic operators, Carnot groups, fundamental solution

\section{Introduction}

Let $\mathbb{G}$ be a Carnot group of homogeneous dimension $Q$ (i.e. a connected and simply connected nilpotent stratified Lie group; see below for precise definitions) identified with $\mathbb{R}^{n}$ through the exponential map, and let $\mathcal{L}$ be a homogeneous left invariant differential operator on $\mathbb{G}$ of order $a=2 r, r \in \mathbb{N}$, with ${ }^{t} \mathcal{L}=\mathcal{L} \geq 0$. Denote by $W_{1}, \ldots, W_{m}$ a basis of the first layer (the horizontal layer) of the Lie algebra $\mathfrak{g}$ of $\mathbb{G}$. The following equivalence is known ([9. Theorem 2.1 and following remark], and also [16, Proposition 1.4.7], for vector bundles over Heisenberg groups).

Theorem 1.1. The following statements are equivalent:

(i) $\mathcal{L}$ is hypoelliptic.

(ii) If $\Omega \subset \mathbb{G}$ is a bounded open set, then there exists $C=C_{\Omega}$ such that for any homogeneous polynomial $P$ in $W_{1}, \ldots, W_{m}$ of degree a we have

$$
\|P \alpha\|_{L^{2}(\mathbb{G})} \leq C\left(\|\mathcal{L} \alpha\|_{L^{2}(\mathbb{G})}+\|\alpha\|_{L^{2}(\mathbb{G})}\right) \quad \text { for any } \alpha \in \mathcal{D}(\Omega),
$$

i.e. $\mathcal{L}$ is maximal hypoelliptic in the sense of [9].

A. Baldi, B. Franchi, M. C. Tesi: Dipartimento di Matematica, Università di Bologna, Piazza di Porta S. Donato 5, 40126 Bologna, Italy; e-mail: baldi@dm.unibo.it, franchib@dm.unibo.it, tesi@dm.unibo.it

Mathematics Subject Classification (2000): 35H05, 35H20, 35B05, 43A80 
More generally, consider now an $N \times N$ matrix-valued operator $\mathcal{L}={ }^{t} \mathcal{L} \geq 0$ of order $a \leq Q$. The aim of this paper is to investigate the relationship between hypoellipticity of $\mathcal{L}$, maximal hypoellipticity, $L^{p}$-maximal hypoellipticity (i.e. sharp $L^{p}$ estimates in appropriate Sobolev spaces for $1<p<\infty)$, and what we call maximal subellipticity (which is basically a sharp high order energy estimate). The equivalence of hypoellipticity, maximal hypoellipticity (and Rockland's condition) can be obtained by repeating verbatim the arguments for scalar operators of Theorem 2.1 in [9]. On the other hand, the proof that hypoellipticity implies $L^{p}$-maximal hypoellipticity requires an argument based on the existence of a both left and right inverse $\mathcal{K}$ for $\mathcal{L}$ (see [6]), as well as precise $L^{p}$ estimates for $\mathcal{K}$.

Indeed, once it is proved that there exists a (matrix-valued) fundamental solution $\Gamma$ for $\mathcal{L}$, and that $\Gamma$, by convolution, defines an operator $\mathcal{K}$ that is both a left and a right inverse of $\mathcal{L}$, the proof of the $L^{p}$-maximal hypoellipticity of $\mathcal{L}$ can be carried out by quite standard arguments.

As in [18], in this paper we prove first the existence of a global fundamental solution $\Gamma$ by adapting the arguments of Folland's proof given for the scalar case when $a<Q$. To cover also the case $a=Q$ (which is crucial for instance when dealing with the fourth order contact Laplacian on intrinsic 1-forms-see e.g. [19], [1]-in the first Heisenberg group where $Q=4$ ), we need to slightly modify the argument to take into account the logarithmic behavior of $\Gamma$.

Arguing as in [6], it is easy to see that $\Gamma$ yields by convolution a right inverse for $\mathcal{L}$.

On the contrary, our proof that the convolution with $\Gamma$ provides a left inverse is carried out in a different way: if $\alpha$ is a compactly supported (say) smooth vector field, then we show that $\beta:=\mathcal{K} \mathcal{L} \alpha-\alpha$ is $\mathcal{L}$-harmonic (i.e. $\mathcal{L} \beta=0$ ) and it vanishes at infinity when $a<Q$, and is bounded when $a=Q$. Then we reach our desired conclusion by relying on a Liouville type theorem for general matrix-valued left invariant homogeneous hypoelliptic operators (inspired by [14]). In other words, unlike [6], we do not use any symmetry property of the fundamental solution. Remember we are dealing with higher order differential operators for which neither an explicit form of $\mathcal{K}$ is known, nor can we easily prove any symmetry property of the fundamental solution with respect to the map $p \mapsto p^{-1}$. In fact, Folland's proof (Corollary 2.8 of [6]) seems to rely heavily on the assumption that $\Gamma\left(p^{-1}\right) \equiv \Gamma(p)$. On the other hand, this symmetry property appears in our proof (when $a<Q$ ) as a consequence of the existence of a left inverse.

In a sense, this approach is akin to that given in [17] and [3, Chapter V, Section 3], when $\mathcal{L}$ is a sum-of-squares operator, where the proof uses a maximum principle. However, clearly, the maximum principle fails to hold for vector-valued functions. We can overcome this difficulty precisely by means of our Liouville type theorem for vectorvalued functions on homogeneous groups (see Proposition 3.2).

In addition, in Theorem 4.1, by an interpolation argument, we prove that the maximal hypoellipticity of $\mathcal{L}$ implies its maximal subellipticity, i.e. if $\Omega \subset \mathbb{G}$ is a bounded open set, then there exists $C=C_{\Omega}$ such that for any homogeneous polynomial $P$ in $W_{1}, \ldots, W_{m}$ of degree $r$ we have

$$
\|P \alpha\|_{L^{2}(\mathbb{G})} \leq C\left(\langle\mathcal{L} \alpha, \alpha\rangle_{L^{2}(\mathbb{G})}+\|\alpha\|_{L^{2}(\mathbb{G})}\right) \quad \text { for any } \alpha \in \mathcal{D}(\Omega)
$$

(see also [9, Chapter III, 7]). 
Finally, we complete this circle of statements by showing that maximal subellipticity implies hypoellipticity. This implication for sum-of-squares operators (of order 2) goes back to [10] and [13]. Our proof is carried out in the spirit of the pseudodifferential arguments given in [15], and consists in a careful representation of the commutator $\left[W^{I}, p(x, D)\right]$ of a homogeneous polynomial $W^{I}$ of degree $|I|$ in the horizontal vector fields $W_{1}, \ldots, W_{m}$ (more precisely, see (11) for this notation), and of a pseudodifferential operator $p(x, D)$ belonging to the usual Hörmander classes (see Lemmata 5.1 and 5.2). In fact, the proof in [10] and [13] for operators of order 2 cannot be repeated here since 2 is a privileged number: indeed, if $W_{i}$ is a horizontal vector field and $p(x, D)$ is a pseudodifferential operator of order 0 , then $\left[W_{i}^{2}, p(x, D)\right] \alpha=q(x, D) W_{i} \alpha+r(x, D) \alpha$, with both $q$ and $r$ of order 0 . Thus, basically, the commutator can be controlled by all the horizontal derivatives of $\alpha$, which in turn can be controlled by $\langle\mathcal{L} \alpha, \alpha\rangle$, by subellipticity (1), i.e. by horizontal derivatives of order half the order of $\mathcal{L}$. In other words, it is crucial that $2-1=\frac{1}{2} 2$. Clearly, this argument fails to work when $a$, the order of $\mathcal{L}$, exceeds 2 . Assuming maximal hypoellipticity instead of maximal subellipticity, i.e. assuming that $\|\mathcal{L} u\|$ controls all the derivatives up to the order of $\mathcal{L}$, this problem is bypassed in [15] thanks to Lemma 5.1, expressing $\left[W^{I}, p(x, D)\right]$ as a sum of terms of the form $q(x, D) W^{J}$, with $|J| \leq|I|-1$. To overcome the problem in our situation, we need a more sophisticated result (Lemma 5.2), where, integrating repeatedly by parts, we can successively distribute in an appropriate way the horizontal derivatives on both sides of the scalar product, so that only horizontal derivatives of order not exceeding $\frac{1}{2} a$ appear.

In this paper, we focus on matrix-valued differential operators, since, typically, this situation arises from a (global) trivialization of a vector fiber bundle $(\mathbb{G}, \mathcal{F}, \pi)$ that is invariant with respect to group translations and dilations. A remarkable example of this situation is provided by the intrinsic Laplacian of the contact complex of Heisenberg groups ([19], [20]). In this context, Theorem 4.1 improves in particular Theorem 4.7 of [1] and the results of [2].

\section{Notations and preliminary results}

A Carnot group $\mathbb{G}$ of step $\kappa$ is a connected, simply connected Lie group whose Lie algebra $\mathfrak{g}$ admits a step $\kappa$ stratification, i.e. there exist linear subspaces $V_{1}, \ldots, V_{\kappa}$ such that

$$
\mathfrak{g}=V_{1} \oplus \cdots \oplus V_{\kappa}, \quad\left[V_{1}, V_{i}\right]=V_{i+1}, \quad V_{\kappa} \neq\{0\}, \quad V_{i}=\{0\} \text { if } i>\kappa,
$$

where $\left[V_{1}, V_{i}\right]$ is the subspace of $\mathfrak{g}$ generated by the commutators $[X, Y]$ with $X \in V_{1}$ and $Y \in V_{i}$. Let $m_{i}=\operatorname{dim}\left(V_{i}\right)$ for $i=1, \ldots, \kappa$ and $h_{i}=m_{1}+\cdots+m_{i}$ with $h_{0}=0$ and, clearly, $h_{\kappa}=n$. Choose a basis $e_{1}, \ldots, e_{n}$ of $\mathfrak{g}$ adapted to the stratification, that is, such that

$$
e_{h_{j-1}+1}, \ldots, e_{h_{j}} \text { is a base of } V_{j} \text { for each } j=1, \ldots, k \text {. }
$$

Let $W=W_{1}, \ldots, W_{n}$ be the family of left invariant vector fields such that $W_{i}(0)=e_{i}$. Given (1), the subset $W_{1}, \ldots, W_{m_{1}}$ generates by commutations all the other vector fields; we will refer to $W_{1}, \ldots, W_{m_{1}}$ as generating vector fields of the group. The exponential 
map is a one-to-one map from $\mathfrak{g}$ onto $\mathbb{G}$, i.e. any $p \in \mathbb{G}$ can be written in a unique way as $p=\exp \left(p_{1} W_{1}+\cdots+p_{n} W_{n}\right)$. Using these exponential coordinates, we identify $p$ with the $n$-tuple $\left(p_{1}, \ldots, p_{n}\right) \in \mathbb{R}^{n}$ and we identify $\mathbb{G}$ with $\left(\mathbb{R}^{n}, \cdot\right)$ where the explicit expression of the group operation - is determined by the Campbell-Hausdorff formula (see [6]); some of its features are described in Proposition 2.1] below. If $p \in \mathbb{G}$ and $i=1, \ldots, \kappa$, we put $p^{i}=\left(p_{h_{i-1}+1}, \ldots, p_{h_{i}}\right) \in \mathbb{R}^{m_{i}}$, so that we can also identify $p$ with $\left[p^{1}, \ldots, p^{\kappa}\right] \in \mathbb{R}^{m_{1}} \times \cdots \times \mathbb{R}^{m_{\kappa}}=\mathbb{R}^{n}$.

The subbundle of the tangent bundle $T \mathbb{G}$ that is spanned by the vector fields $W_{1}, \ldots$, $W_{m_{1}}$ plays a particularly important role in the theory, it is called the horizontal bundle $H \mathbb{G}$; the fibers of $H \mathbb{G}$ are

$$
H \mathbb{G}_{x}=\operatorname{span}\left\{W_{1}(x), \ldots, W_{m_{1}}(x)\right\}, \quad x \in \mathbb{G} .
$$

From now on, for simplicity, we sometimes set $m:=m_{1}$.

A subriemannian structure is defined on $\mathbb{G}$, endowing each fiber of $H \mathbb{G}$ with a scalar product $\langle\cdot, \cdot\rangle_{x}$ and a norm $|\cdot|_{x}$ that make $W_{1}(x), \ldots, W_{m}(x)$ an orthonormal basis. That is, if $v=\sum_{i=1}^{m} v_{i} W_{i}(x)=\left(v_{1}, \ldots, v_{m}\right)$ and $w=\sum_{i=1}^{m} w_{i} W_{i}(x)=\left(w_{1}, \ldots, w_{m}\right)$ are in $H \mathbb{G}_{x}$, then $\langle v, w\rangle_{x}:=\sum_{j=1}^{m} v_{j} w_{j}$ and $|v|_{x}^{2}:=\langle v, v\rangle_{x}$. The sections of $H \mathbb{G}$ are called horizontal sections, and each vector of $H \mathbb{G}_{x}$ is a horizontal vector.

Two important families of automorphisms of $\mathbb{G}$ are the group translations and the group dilations of $\mathbb{G}$. For any $x \in \mathbb{G}$, the (left) translation $\tau_{x}: \mathbb{G} \rightarrow \mathbb{G}$ is defined as

$$
z \mapsto \tau_{x} z:=x \cdot z .
$$

For any $\lambda>0$, the dilation $\delta_{\lambda}: \mathbb{G} \rightarrow \mathbb{G}$ is defined as

$$
\delta_{\lambda}\left(x_{1}, \ldots, x_{n}\right)=\left(\lambda^{d_{1}} x_{1}, \ldots, \lambda^{d_{n}} x_{n}\right),
$$

where $d_{i} \in \mathbb{N}$ is called the homogeneity of the variable $x_{i}$ in $\mathbb{G}$ (see [7, Chapter 1]) and is defined as

$$
d_{j}=i \quad \text { whenever } \quad h_{i-1}+1 \leq j \leq h_{i},
$$

hence $1=d_{1}=\cdots=d_{m_{1}}<d_{m_{1}+1}=2 \leq \cdots \leq d_{n}=\kappa$.

In the following propositions we collect some more or less elementary properties of the group operation and of the canonical vector fields (see e.g. [8, Propositions 2.1 and 2.2]).

Proposition 2.1. The group product has the form

$$
x \cdot y=x+y+\mathcal{Q}(x, y), \quad \forall x, y \in \mathbb{R}^{n} .
$$

where $\mathcal{Q}=\left(\mathcal{Q}_{1}, \ldots, \mathcal{Q}_{n}\right): \mathbb{R}^{n} \times \mathbb{R}^{n} \rightarrow \mathbb{R}^{n}$ and each $\mathcal{Q}_{i}$ is a homogeneous polynomial of degree $d_{i}$ with respect to the intrinsic dilations of $\mathbb{G}$ defined in (2), that is,

$$
\mathcal{Q}_{i}\left(\delta_{\lambda} x, \delta_{\lambda} y\right)=\lambda^{d_{i}} \mathcal{Q}_{i}(x, y), \quad \forall x, y \in \mathbb{G} .
$$

Moreover, again for all $x, y \in \mathbb{G}$,

$$
\begin{aligned}
& \mathcal{Q}_{1}(x, y)=\cdots=\mathcal{Q}_{m_{1}}(x, y)=0, \\
& \mathcal{Q}_{j}(x, 0)=\mathcal{Q}_{j}(0, y)=0 \quad \text { and } \quad \mathcal{Q}_{j}(x, x)=\mathcal{Q}_{j}(x,-x)=0, \quad \text { for } m_{1}<j \leq n, \\
& \mathcal{Q}_{j}(x, y)=\mathcal{Q}_{j}\left(x_{1}, \ldots, x_{h_{i-1}}, y_{1}, \ldots, y_{h_{i-1}}\right) \quad \text { if } 1<i \leq \kappa \text { and } j \leq h_{i} .
\end{aligned}
$$


Note that from Proposition 2.1 it follows that

$$
\delta_{\lambda} x \cdot \delta_{\lambda} y=\delta_{\lambda}(x \cdot y)
$$

and that the inverse $x^{-1}$ of an element $x=\left(x_{1}, \ldots, x_{n}\right) \in\left(\mathbb{R}^{n}, \cdot\right)$ has the form

$$
x^{-1}=\left(-x_{1}, \ldots,-x_{n}\right)
$$

(see [7, Proposition 2.1] and also [12]).

Proposition 2.2. The vector fields $W_{j}$ have polynomial coefficients and have the form

$$
W_{j}=\partial_{j}+\sum_{i>h_{l}}^{n} q_{i, j}(x) \partial_{i} \quad \text { for } j=1, \ldots, n \text { and } j \leq h_{l}
$$

where $q_{i, j}(x)=\left.\frac{\partial \mathcal{Q}_{i}}{\partial y_{j}}(x, y)\right|_{y=0}$ so that if $j \leq h_{l}$ then $q_{i, j}(x)=q_{i, j}\left(x_{1}, \ldots, x_{h_{l-1}}\right)$ and $q_{i, j}(0)=0$.

In particular, the generating vector fields are homogeneous of degree 1 with respect to group dilations.

In this paper we denote by $\rho$ a homogeneous norm, smooth outside the origin, that induces a genuine distance on $\mathbb{G}$ as in [21, p. 638]. Later on, we shall use the following gauge distance:

$$
d(x, y):=\rho\left(y^{-1} \cdot x\right) .
$$

We shall denote by $U(p, r)$ and $B(p, r)$ respectively the open and closed balls associated with $d$.

The metric $d$ is well behaved with respect to left translations and dilations, that is,

$$
d(z \cdot x, z \cdot y)=d(x, y), \quad d\left(\delta_{\lambda}(x), \delta_{\lambda}(y)\right)=\lambda d(x, y)
$$

for $x, y, z \in \mathbb{G}$ and $\lambda>0$.

The integer

$$
Q=\sum_{j=1}^{n} d_{j}=\sum_{i=1}^{\kappa} i \operatorname{dim} V_{i}
$$

is the homogeneous dimension of $\mathbb{G}$. It is also the Hausdorff dimension of $\mathbb{R}^{n}$ with respect to $d$.

The $n$-dimensional Lebesgue measure $\mathcal{L}^{n}$ is the Haar measure of the group $\mathbb{G}$. Hence if $E \subset \mathbb{R}^{n}$ is measurable, then $\mathcal{L}^{n}(x \cdot E)=\mathcal{L}^{n}(E)$ for all $x \in \mathbb{G}$. Moreover, if $\lambda>0$ then $\mathcal{L}^{n}\left(\delta_{\lambda}(E)\right)=\lambda^{Q} \mathcal{L}^{n}(E)$. We explicitly observe that

$$
\mathcal{L}^{n}(U(p, r))=r^{Q} \mathcal{L}^{n}(U(p, 1))=r^{Q} \mathcal{L}^{n}(U(0,1)) .
$$

All the spaces $L^{p}(\mathbb{G})$ that appear throughout this paper are defined with respect to the $\mathcal{L}^{n}$ Lebesgue measure. 
Following e.g. [7], we can define a group convolution in $\mathbb{G}$ : if, for instance, $f \in \mathcal{D}(\mathbb{G})$ and $g \in L_{\text {loc }}^{1}(\mathbb{G})$, we set

$$
f * g(p):=\int f(q) g\left(q^{-1} p\right) d q \text { for } p \in \mathbb{G} .
$$

We recall that, if (say) $g$ is a smooth function and $L$ is a left invariant differential operator, then $L(f * g)=f * L g$. We also recall that the convolution is again well defined when $f, g \in \mathcal{D}^{\prime}(\mathbb{G})$, provided at least one of them has compact support (as is customary, we denote by $\mathcal{E}^{\prime}(\mathbb{G})$ the class of compactly supported distributions in $\mathbb{G}$ identified with $\mathbb{R}^{n}$, the dual of $\mathcal{E}(\mathbb{G})$, the space of smooth functions). We recall now the notion of kernel of $\operatorname{order} \alpha$. Following [6], a kernel of order $\alpha$ is a homogeneous distribution of degree $\alpha-Q$ (with respect to group dilations $\delta_{r}$ as in (2), see [7]) that is smooth outside the origin.

Following [7], we also adopt the following multi-index notation for higher order derivatives. If $I=\left(i_{1}, \ldots, i_{n}\right)$ is a multi-index, we set

$$
W^{I}=W_{1}^{i_{1}} \cdots W_{n}^{i_{n}}
$$

By the Poincaré-Birkhoff-Witt theorem (see, e.g. [4, I.2.7]), the differential operators $W^{I}$ form a basis for the algebra of left invariant differential operators in $\mathbb{G}$. Furthermore, we set $|I|:=i_{1}+\cdots+i_{n}$, the order of the differential operator $W^{I}$, and $d(I):=d_{1} i_{1}+$ $\cdots+d_{n} i_{n}$, its degree of homogeneity with respect to group dilations. From the PoincaréBirkhoff-Witt theorem it follows in particular that any homogeneous linear differential operator in the horizontal derivatives can be expressed as a linear combination of the operators $W^{I}$ of the special form above. Thus, often we can restrict ourselves to consider only operators of the special form $W^{I}$. Let $k$ be a positive integer, $1 \leq p<\infty$, and $\Omega$ be an open set in $\mathbb{G}$. The Folland-Stein Sobolev space $W_{\mathbb{G}}^{k, p}(\Omega)$ associated with the vector fields $W_{1}, \ldots, W_{n}$ is defined to consist of all functions $f \in L^{p}(\Omega)$ with distributional derivatives $W^{I} f \in L^{p}(\Omega)$ for any $W^{I}$ as above with $d(I) \leq k$, endowed with the natural norm. We keep the subscript $\mathbb{G}$ to avoid misunderstanding with the usual Sobolev spaces $W^{k, p}(\Omega)$. Moreover, we denote by $\stackrel{\circ}{W}_{\mathbb{G}}^{k, p}(\Omega)$ the completion of $\mathcal{D}(\Omega)$ in $W_{\mathbb{G}}^{k, p}(\Omega)$.

Let $N \in \mathbb{N}$ be given. From now on, we are dealing with $N$-vector-valued functions $\alpha=\left(\alpha_{1}, \ldots, \alpha_{N}\right)$. If $\Omega \subset \mathbb{G}$ is an open set, $k \in \mathbb{N}$ and $1 \leq p \leq \infty$, we still denote by $W_{\mathbb{G}}^{k, p}(\Omega)\left(\right.$ resp. $\left.\stackrel{\circ}{W}_{\mathbb{G}}^{k, p}(\Omega)\right)$ the vector-valued function spaces $\left(W_{\mathbb{G}}^{s, p}(\Omega)\right)^{N}$ (resp. $\left.\left(\stackrel{\circ}{W}_{\mathbb{G}}^{k, p}(\Omega)\right)^{N}\right)$. Analogously, we shall write $L^{p}(\Omega)$ for $\left(L^{p}(\Omega)\right)^{N}$ and $W^{k, p}(\Omega)$ for $\left(W^{k, p}(\Omega)\right)^{N}$.

Moreover, if $T=\left(T_{1}, \ldots, T_{N}\right) \in \mathcal{D}^{\prime}(\Omega)^{N}$, then $T$ can be identified with an element of the dual of $\mathcal{D}\left(\Omega, \mathbb{R}^{N}\right)$ acting as

$$
\langle T \mid \phi\rangle:=\sum_{j}\left\langle T_{j} \mid \phi_{j}\right\rangle
$$

for $\phi=\left(\phi_{1}, \ldots, \phi_{N}\right) \in \mathcal{D}\left(\Omega, \mathbb{R}^{N}\right)$. The same convention holds for $T=\left(T_{1}, \ldots, T_{N}\right) \in$ $\mathcal{S}^{\prime}(\Omega)^{N}$. 
We recall that, if $T=\left(T_{1}, \ldots, T_{N}\right) \in \mathcal{S}^{\prime}(\mathbb{G})^{N}$, then there exist $k, h \in \mathbb{N} \cup\{0\}$ and $C(T)>0$ such that

$$
\left|\left\langle T_{j} \mid \phi\right\rangle\right| \leq C(T)\|\phi\|_{k, h} \quad \text { for all } \phi \in \mathcal{S}\left(\mathbb{G}, \mathbb{R}^{N}\right) \text { and } j=1, \ldots, N,
$$

where, with the standard notation for multi-indices,

$$
\|\phi\|_{k, h}=\max _{|\alpha| \leq k,|\beta| \leq h} \sup _{p \in \mathbb{G}}\left|p^{\alpha} \partial^{\beta} \phi(p)\right| .
$$

Hence

$$
|\langle T \mid \phi\rangle| \leq C(T)\|\phi\|_{k, h} \quad \text { for all } \phi \in \mathcal{S}\left(\mathbb{G}, \mathbb{R}^{N}\right) .
$$

Remark 2.3. It is easy to see that the polynomials $q_{i, j}$ of Proposition 2.2 have degree at most $\kappa-1$. Moreover, by (7), $\partial_{j}$ can be written as a sum of the form

$$
\partial_{j}=\sum_{i} r_{i, j}(x) W_{i}
$$

where the $r_{i, j}$ 's are polynomials of degree at most $\kappa-1$, for $j=1, \ldots, n$. Hence, if $\alpha=\left(\alpha_{1}, \ldots, \alpha_{n}\right)$ and $\beta=\left(\beta_{1}, \ldots, \beta_{n}\right)$ are multi-indices, then $x^{\alpha} \partial^{\beta}$ can be written as a sum of the form

$$
x^{\alpha} \partial^{\beta}=\sum_{|\gamma| \leq(\kappa-1)|\beta|+|\alpha|,|I| \leq|\beta|} c_{\gamma, I} x^{\gamma} W^{I}
$$

with $c_{\gamma, I}$ suitable real constants.

\section{Liouville type theorems and fundamental solution}

Let $\mathcal{L}:=\left(\mathcal{L}_{j i}\right)_{j, i=1, \ldots, N}$ be a differential operator on $\mathcal{E}\left(\mathbb{G}, \mathbb{R}^{N}\right)$ defined by

$$
\mathcal{L}\left(\alpha_{1}, \ldots, \alpha_{N}\right)=\left(\sum_{i} \mathcal{L}_{i 1} \alpha_{i}, \ldots, \sum_{i} \mathcal{L}_{i N} \alpha_{i}\right),
$$

where the $\mathcal{L}_{i j}$ 's are constant coefficient homogeneous polynomials of degree $a$ in $W_{1}, \ldots$, $W_{m}$. Due to the left invariance and the homogeneity (with respect to group dilations) of the vector fields $W_{1}, \ldots, W_{m}$, we say that the operator $\mathcal{L}$ is left invariant and homogeneous of degree $a$. We notice that the formal adjoint ${ }^{t} \mathcal{L}$ of $\mathcal{L}$ is given by

$$
{ }^{t} \mathcal{L}\left(\alpha_{1}, \ldots, \alpha_{N}\right)=\left(\sum_{i}{ }^{t} \mathcal{L}_{1 i} \alpha_{i}, \ldots, \sum_{i}{ }^{t} \mathcal{L}_{N i} \alpha_{i}\right) .
$$

In this section we proove a Liouville type theorem and study the (matrix-valued) fundamental solution for $\mathcal{L}$. In particular, these results improve Theorem 4.7 and Proposition 4.10 of [1], and will be used later to derive sharp $L^{p}$ estimates for the operator $\mathcal{L}$ in Theorem 4.1 The role of the Liouville theorem as well as the relationship with Folland's construction of the fundamental solution have already been extensively discussed in the Introduction. 
Theorem 3.1. Suppose $\mathcal{L}$ is a left invariant hypoelliptic differential operator such that ${ }^{t} \mathcal{L}=\mathcal{L}$. Suppose also that $\mathcal{L}$ is homogeneous of degree $a \leq Q$. Then there exist

$$
K_{j}=\left(K_{1 j}, \ldots, K_{N j}\right), \quad j=1, \ldots, N,
$$

with $K_{i j} \in \mathcal{D}^{\prime}(\mathbb{G}) \cap \mathcal{E}(\mathbb{G} \backslash\{0\}), i, j=1, \ldots, N$, such that:

(i) We have

$$
\sum_{i} \mathcal{L}_{i \ell} K_{i j}= \begin{cases}\delta & \text { if } \ell=j \\ 0 & \text { if } \ell \neq j\end{cases}
$$

(ii) If $a<Q$, then the $K_{i j}$ 's are kernels of type $a$ in the sense of [6], for $i, j=1, \ldots, N$ (i.e. they are smooth functions outside of the origin, homogeneous of degree $a-Q$, and hence belonging to $L_{\mathrm{loc}}^{1}(\mathbb{G})$, by Corollary 1.7 of [6]). If $a=Q$, then the $K_{i j}$ 's satisfy the logarithmic estimate $\left|K_{i j}(p)\right| \leq C(1+|\ln \rho(p)|)$ and hence belong to $L_{\text {loc }}^{1}(\mathbb{G})$. Moreover, their horizontal derivatives (i.e. $W_{\ell} K_{i j}$ for $\left.\ell=1, \ldots, m\right)$ are kernels of type $Q-1$ in the sense of [6].

(iii) When $\alpha \in \mathcal{D}\left(\mathbb{G}, \mathbb{R}^{N}\right)$, if we set

$$
\mathcal{K} \alpha:=\left(\sum_{j} \alpha_{j} * K_{1 j}, \ldots, \sum_{j} \alpha_{j} * K_{N j}\right),
$$

then $\mathcal{L} \mathcal{K} \alpha=\alpha$. Moreover, if $a<Q$, also $\mathcal{K} \mathcal{L} \alpha=\alpha$.

(iv) If $a=Q$, then for any $\alpha \in \mathcal{D}\left(\mathbb{G}, \mathbb{R}^{N}\right)$ there exists $\beta_{\alpha}:=\left(\beta_{1}, \ldots, \beta_{N}\right) \in \mathbb{R}^{N}$ such that

$$
\mathcal{K} \mathcal{L} \alpha-\alpha=\beta_{\alpha} .
$$

Proof. The content of the theorem is similar to that of Theorem 4.7 and Proposition 4.10 of [1] (stated there in terms of forms and currents on the contact complex of Heisenberg groups), except for the (non-trivial) fact that here we are assuming $\mathcal{L}$ is merely hypoelliptic and not maximal hypoelliptic as in [1]. We stress that maximal hypoellipticity is used in the proofs of Theorem 4.7 and Proposition 4.10 of [1] only to show that a smooth intrinsic form $\omega$ in the contact complex (which can be identified in the context of the present note with a vector-valued smooth function $\omega=\left(\omega_{1}, \ldots, \omega_{N}\right)$ for a suitable $\left.N \in \mathbb{N}\right)$ that satisfies

(a) if $a<Q$ and $R$ is a homogeneous differential operator in the horizontal vector fields of degree $\ell<a$, then

$$
R \omega(p)=O\left(\rho(p)^{a-\ell-Q}\right) \text { as } p \rightarrow \infty,
$$

(b) if $a=Q$ and $R$ is a homogeneous differential operator in the horizontal vector fields of degree $\ell<a$, then

$$
\begin{array}{lll}
R \omega(p)=O\left(\rho(p)^{-\ell}\right) & \text { as } p \rightarrow \infty & \text { if } \ell>0 \\
R \omega(p)=O(\ln \rho(p)) & \text { as } p \rightarrow \infty & \text { if } \ell=0
\end{array}
$$

(c) $\mathcal{L} \omega=0$ 
has necessarily polynomial coefficients and hence vanishes identically if $a<Q$ or has constant coefficients if $a=Q$. Inspired by [14], we are able to prove the same statement as a consequence of a Liouville type theorem (see Proposition 3.2 below), under the only assumption that $\mathcal{L}$ is homogeneous, left invariant and hypoelliptic.

Thus, the proof of Theorem 3.1 will be completed by proving the following result.

Proposition 3.2. If $T=\left(T_{1}, \ldots, T_{N}\right) \in \mathcal{S}^{\prime}(\mathbb{G})^{N}$ satisfies $\mathcal{L} T=0$, then $T$ is a (vectorvalued) polynomial.

In order to prove Proposition 3.2 , let us start with the following lemma.

Lemma 3.3 (cf. [14, Lemma 4]). Let $\mathcal{L}$ be as in Theorem 3.1. If $T=\left(T_{1}, \ldots, T_{N}\right) \in$ $\mathcal{S}^{\prime}(\mathbb{G})^{N}$ and $\mathcal{L} T=0$, then there exists a constant $M=M(a, T)$ such that $W^{I} T_{j}(0)=0$ when $|I|>M$ (remember we can assume the $T_{j}$ 's are smooth functions, by hypoellipticity). More precisely, the constant $M$ depends on the homogeneity degree a of $\mathcal{L}$ and on the indices $k, h$ of the seminorm $\|\cdot\|_{k, h}$ associated with $T$ as in (12).

Proof. By (12), there exist $k, h \in \mathbb{N} \cup\{0\}$ such that

$$
\left\langle T_{j} \mid \phi\right\rangle \leq C(T)\|\phi\|_{k, h} \quad \text { for all } \phi \in \mathcal{S}(\mathbb{G}) \text { and } j=1, \ldots, N .
$$

If $\phi \in \mathcal{S}(\mathbb{G})$, then, with our notations, if $r \geq 1$, we have $\phi \circ \delta_{1 / r} \in \mathcal{S}(\mathbb{G})$ and

$$
\left\|\phi \circ \delta_{1 / r}\right\|_{k, h} \leq r^{Q k}\|\phi\|_{k, h} .
$$

Let now $I:=\left(i_{1}, \ldots, i_{N}\right)$ be a multi-index. If $M=(k-1) Q$ the integral

$$
\left\langle F_{I} \mid \phi\right\rangle:=\int_{1}^{\infty} r^{-Q-d(I)-1}\left\langle T \mid \phi \circ \delta_{1 / r}\right\rangle d r
$$

is well defined for any $\phi \in \mathcal{S}\left(\mathbb{G}, \mathbb{R}^{N}\right)$ when $|I|>M$. Indeed, since $d(I) \geq|I|$, and by (20) and (21), we have

$$
\begin{aligned}
\left|\left\langle F_{I} \mid \phi\right\rangle\right| & \leq C(T)\|\phi\|_{k, h} \int_{1}^{\infty} r^{-Q-d(I)-1+k Q} d r \\
& \leq C(T)\|\phi\|_{k, h} \int_{1}^{\infty} r^{-Q-|I|-1+k Q} d r=\frac{1}{|I|-M} C(T)\|\phi\|_{k, h} .
\end{aligned}
$$

In particular, we notice that $F_{I} \in \mathcal{S}^{\prime}(\mathbb{G})^{N}$ whenever $|I|>M$. Next, we prove that $\mathcal{L} F_{I}=0$ when $|I|>M$. Clearly,

$$
\begin{aligned}
\left\langle\mathcal{L} F_{I} \mid \phi\right\rangle & =\left\langle\left. F_{I}\right|^{t} \mathcal{L} \phi\right\rangle=\left\langle F_{I} \mid \mathcal{L} \phi\right\rangle=\int_{1}^{\infty} r^{-Q-d(I)-1}\left\langle T \mid(\mathcal{L} \phi) \circ \delta_{1 / r}\right\rangle d r \\
& =\int_{1}^{\infty} r^{-Q-d(I)-1+a}\left\langle T \mid \mathcal{L}\left(\phi \circ \delta_{1 / r}\right)\right\rangle d r \\
& =\int_{1}^{\infty} r^{-Q-d(I)-1+a}\left\langle\mathcal{L} T \mid \phi \circ \delta_{1 / r}\right\rangle d r=0
\end{aligned}
$$


for any $\phi \in \mathcal{S}\left(\mathbb{G}, \mathbb{R}^{N}\right)$. Hence $\mathcal{L} F_{I}=0$ and thus $F_{I}=:\left(F_{I, 1}, \ldots, F_{I, N}\right)$ is smooth, by hypoellipticity, provided $|I|>M$. Choose now a scalar mollifier $\psi \in \mathcal{D}(\mathbb{G})$ such that $\psi \geq 0$ and $\int \psi(p) d p=1$. We set $\psi_{\varepsilon}:=\varepsilon^{-Q} \psi \circ \delta_{1 / \varepsilon}, \phi_{\varepsilon}=\phi_{j, \varepsilon}=\psi_{\varepsilon} e_{j}$ with $1 \leq j \leq N$, where $e_{j}$ is the $j$-th vector of the canonical orthonormal basis of $\mathbb{R}^{N}$. Thus

$$
W^{I} F_{I, j}(0)=\lim _{\varepsilon \rightarrow 0}\left\langle W^{I} F_{I} \mid \phi_{\varepsilon}\right\rangle
$$

for $j=1, \ldots, N$, provided $|I|>M$. On the other hand, always when $|I|>M$,

$$
\begin{aligned}
\left\langle W^{I} F_{I} \mid \phi_{\varepsilon}\right\rangle & =(-1)^{|I|}\left\langle F_{I} \mid W^{I} \phi_{\varepsilon}\right\rangle=(-1)^{|I|} \int_{1}^{\infty} r^{-Q-d(I)-1}\left\langle T \mid\left(W^{I} \phi_{\varepsilon}\right) \circ \delta_{1 / r}\right\rangle d r \\
& =(-1)^{|I|} \int_{1}^{\infty} r^{-Q-1}\left\langle T \mid W^{I}\left(\phi_{\varepsilon} \circ \delta_{1 / r}\right)\right\rangle d r \\
& =\int_{1}^{\infty} r^{-Q-1}\left\langle W^{I} T \mid \phi_{\varepsilon} \circ \delta_{1 / r}\right\rangle d r=\int_{1}^{\infty} r^{-Q-1}\left\langle W^{I} T_{j} \mid \psi_{\varepsilon} \circ \delta_{1 / r}\right\rangle d r \\
& =\varepsilon^{-Q} \int_{1}^{\infty} r^{-Q-1}\left\langle W^{I} T_{j} \mid \psi \circ \delta_{1 /(\varepsilon r)}\right\rangle d r \\
& =\int_{\varepsilon}^{\infty} \lambda^{-Q-1}\left\langle W^{I} T_{j} \mid \psi \circ \delta_{1 / \lambda}\right\rangle d \lambda=\int_{\varepsilon}^{\infty} \lambda^{-1}\left\langle W^{I} T_{j} \mid \psi_{\lambda}\right\rangle d \lambda .
\end{aligned}
$$

Then, using also [23, we get

$$
W^{I} F_{I, j}(0)=\int_{0}^{\infty} \lambda^{-1}\left\langle W^{I} T_{j} \mid \psi_{\lambda}\right\rangle d \lambda
$$

for $j=1, \ldots, N$, provided $|I|>M$. Since $T$ is smooth as remarked above, $\left\langle W^{I} T_{j} \mid \psi_{\lambda}\right\rangle$ $\rightarrow W^{I} T_{j}(0)$ as $\lambda \rightarrow 0$. Now, since the integral in (24) is convergent because the left-hand side of [24] is finite, $W^{I} T_{j}(0)$ must be equal to zero for any $I$ such that $|I|>M$; this holds for any $j=1, \ldots, N$. Therefore, $W^{I} T=0$ for any $I$ such that $|I|>M$, and the proof is complete.

Proof of Proposition 3.2 By hypoellipticity, $T$ is smooth. Let now $q \in \mathbb{G}$ be fixed. We want to apply Lemma 3.3 to $T \circ \tau_{q}$. In fact, suppose we have shown that, if

$$
|\langle T \mid \phi\rangle| \leq C(T)\|\phi\|_{k, h} \quad \text { for all } \phi \in S\left(\mathbb{G}, \mathbb{R}^{N}\right),
$$

for given $k, h \in \mathbb{N}$, then also

$$
\left|\left\langle T \circ \tau_{q} \mid \phi\right\rangle\right| \leq C(q, T)\|\phi\|_{k^{\prime}, h^{\prime}} \quad \text { for all } \phi \in S\left(\mathbb{G}, \mathbb{R}^{N}\right),
$$

with $h^{\prime}, k^{\prime}$ depending only on the structure constants of $\mathbb{G}$ appearing in Propositions 2.1 and 2.2 and on $h, k$ of 25, but not on $q$. Since $\mathcal{L}$ is invariant under group translations, we have $\mathcal{L}\left(T \circ \tau_{q}\right)=0$, and eventually, by Lemma 3.3. $W^{I}\left(T \circ \tau_{q}\right)(0)=0$ when $|I|>M$, with $M$ independent of $q \in \mathbb{G}$. But, by the left invariance of $W^{I}$,

$$
W^{I}\left(T \circ \tau_{q}\right)_{j}(0)=W^{I}\left(T_{j} \circ \tau_{q}\right)(0)=\left(\left(W^{I} T_{j}\right) \circ \tau_{q}\right)(0)=W^{I} T_{j}(q),
$$


so that $W^{I} T_{j} \equiv 0$ for $j=1, \ldots, N$ when $|I|>M$. Then all derivatives of $T$ of sufficiently high order vanish identically, and the assertion follows.

Thus, we are left with the proof of 26]. We note first $\left|\left\langle T \circ \tau_{q} \mid \phi\right\rangle\right|=\left|\left\langle T \mid \phi \circ \tau_{q^{-1}}\right\rangle\right|$, and so we only have to show that

$$
\left\|\phi \circ \tau_{q^{-1}}\right\|_{k, h} \leq C(q)\|\phi\|_{k^{\prime}, h^{\prime}} \quad \text { for all } \phi \in \mathcal{S}\left(\mathbb{G}, \mathbb{R}^{N}\right)
$$

with $h^{\prime}, k^{\prime}$ depending only on the structure constants of $\mathbb{G}$ appearing in Propositions 2.1 and 2.2 and on $h, k$ of 25 , but not on $q$.

Without loss of generality, we may restrict ourselves to the case $N=1$. By (15), if $|\alpha| \leq k$ and $|\beta| \leq h,\left|p^{\alpha} \partial^{\beta}\left(\phi \circ \tau_{q^{-1}}\right)(p)\right|$ can be estimated by a sum of terms of the form

$$
\left|p^{\gamma} W^{I}\left(\phi\left(q^{-1} \cdot p\right)\right)\right|=\left|p^{\gamma}\left(W^{I} \phi\right)\left(q^{-1} \cdot p\right)\right|=\left|\left(q \cdot q^{-1} \cdot p\right)^{\gamma}\left(W^{I} \phi\right)\left(q^{-1} \cdot p\right)\right|,
$$

with $|\gamma| \leq(\kappa-1)|\beta|+|\alpha| \leq(\kappa-1) h+k$ and $|I| \leq|\beta| \leq h$. Therefore, by Proposition 2.2 and Remark 2.3. and then by Proposition 2.1.

$$
\begin{aligned}
\sup _{\substack{p \in \mathbb{G},|\alpha| \leq k,|\beta| \leq h}} \| p^{\alpha} \partial^{\beta}\left(\phi \circ \tau_{q^{-1}}\right)(p) \mid & \leq \sup _{\substack{z \in \mathbb{G},|I| \leq h \\
|\gamma| \leq \kappa-1) h+k}}\left|(q \cdot z)^{\gamma}\left(W^{I} \phi\right)(z)\right| \\
& \leq \sup _{\substack{z \in \mathbb{G},|I| \leq h \\
|\gamma| \leq 2(\kappa-1) h+k}}\left|(q \cdot z)^{\gamma}\left(\partial_{z}^{I} \phi\right)(z)\right| \\
& \leq C(q) \sup _{\substack{z \in \mathbb{G},|I| \leq h \\
|\gamma| \leq \kappa(2(\kappa-1) h+k)}}\left|z^{\gamma} \partial_{z}^{I} \phi(z)\right| \\
& =C(q)\|\phi\|_{\kappa(2(\kappa-1) h+k), h}=: C(q)\|\phi\|_{k^{\prime}, h^{\prime}} .
\end{aligned}
$$

\section{Main result}

Theorem 4.1. Let

$$
\mathcal{L}: \mathcal{E}\left(\mathbb{G}, \mathbb{R}^{N}\right) \rightarrow \mathcal{E}\left(\mathbb{G}, \mathbb{R}^{N}\right)
$$

be a self-adjoint non-negative left invariant differential operator that is homogeneous (with respect to group dilations) of degree $a:=2 r \leq Q$, where $r \in \mathbb{N}$ and $Q$ is the homogeneous dimension of $\mathbb{G}$. The following statements are equivalent:

(i) $\mathcal{L}$ is hypoelliptic.

(ii) $\mathcal{L}$ is maximal subelliptic, i.e., if $\Omega \subset \mathbb{G}$ is a bounded open set, then there exists $C=C_{\Omega}$ such that for any multi-index $I$ with $|I|=r$,

$$
\left\|W^{I} \alpha\right\|_{L^{2}(\mathbb{G})} \leq C\left(\langle\mathcal{L} \alpha, \alpha\rangle_{L^{2}(\mathbb{G})}+\|\alpha\|_{L^{2}(\mathbb{G})}^{2}\right)^{1 / 2} \quad \text { for any } \alpha \in \mathcal{D}\left(\Omega, \mathbb{R}^{N}\right) .
$$

(iii) $\mathcal{L}$ is maximal hypoelliptic in the sense of [9], i.e., if $\Omega \subset \mathbb{G}$ is a bounded open set, then there exists $C=C_{\Omega}$ such that for any multi-index $I$ with $|I|=2 r$ we have

$$
\left\|W^{I} \alpha\right\|_{L^{2}(\mathbb{G})} \leq C\left(\|\mathcal{L} \alpha\|_{L^{2}(\mathbb{G})}+\|\alpha\|_{L^{2}(\mathbb{G})}\right) \quad \text { for any } \alpha \in \mathcal{D}\left(\Omega, \mathbb{R}^{N}\right) .
$$


(iv) If $1<p<\infty$ is fixed, and $\Omega \subset \mathbb{G}$ is a bounded open set, then there exists $C=C_{\Omega, p}$ such that for any multi-index $I$ with $|I|=2 r$ we have

$$
\left\|W^{I} \alpha\right\|_{L^{p}(\mathbb{G})} \leq C\left(\|\mathcal{L} \alpha\|_{L^{p}(\mathbb{G})}+\|\alpha\|_{L^{p}(\mathbb{G})}\right) \quad \text { for any } \alpha \in \mathcal{D}\left(\Omega, \mathbb{R}^{N}\right)
$$

By the Poincaré inequality [12], in [30, 29] (and in (28), we can replace $|I|=2 r$ by $|I| \leq 2 r(|I|=r$ by $|I| \leq r$, respectively $)$.

Proof. Clearly, (iv) implies (iii). To show that (iii) yields (ii) we argue as in [5, Theorem 1]. Indeed, by dilation, it is easy to see that the constant $C$ in $(29)$ can be chosen independent of $\Omega$. Hence $(29)$ says that the domain of $\mathcal{L}$ in $L^{2}(\mathbb{G})$ with the graph norm is continuously imbedded in $W_{\mathbb{G}}^{2 r, 2}(\mathbb{G})$. By interpolation, the domain of $\mathcal{L}^{1 / 2}$ with the norm graph is continuously imbedded in $W_{\mathbb{G}}^{r, 2}(\mathbb{G})$ (remember that the interpolation space of order $\frac{1}{2}$ between $W_{\mathbb{G}}^{2 r, 2}(\mathbb{G})$ and $L^{2}(\mathbb{G})$ is $W_{\mathbb{G}}^{r, 2}(\mathbb{G})$, by [6, Theorem 4.10 and Proposition 4.1]). But this is precisely [28).

Let us prove that (ii) implies (i). For notations concerning pseudodifferential operators, see Section 5

Step 1. If $\Omega \subset \mathbb{G}$ is a bounded open set, then there exists $C>0$ such that, denoting by $\|\cdot\|_{s}$ the norm in $W_{\mathbb{G}}^{s, 2}(\mathbb{G})$,

$$
\sum_{|I| \leq r}\left\|W^{I} \alpha\right\|_{\delta(r-|I|)}^{2} \leq C\left(\langle\mathcal{L} \alpha, \alpha\rangle+\|\alpha\|_{0}^{2}\right) \quad \text { for any } \alpha \in \mathcal{D}\left(\Omega, \mathbb{R}^{N}\right) .
$$

We can restrict ourselves to the case $|I|<r$, since, when $|I|=r$, then $W^{I} \alpha$ is already estimated by the right hand side of (31) by (28). Moreover, we shall carry out our computations in the case $|I|>0$. The case $|I|=0$ requires only minor modifications. Set

$$
S_{h}:=\sum_{|J| \leq h}\left\|W^{J} \alpha\right\|_{\delta(r-|J|)}
$$

Notice $S_{h} \leq S_{h+1}$.

For $s \in \mathbb{R}$, denote by $\Lambda^{s}$ the pseudodifferential operator of symbol $\left(1+|\xi|^{2}\right)^{s / 2}$. We still denote by $\Lambda^{s}$ the diagonal operator induced on $\mathcal{D}^{\prime}\left(\Omega, \mathbb{R}^{N}\right)$ as follows: if $T=$ $\left(T_{1}, \ldots, T_{N}\right) \in \mathcal{D}\left(\Omega, \mathbb{R}^{N}\right)$, we write

$$
\Lambda^{s} T:=\left(\Lambda^{s} T_{1}, \ldots, \Lambda^{s} T_{N}\right)
$$

By Lemma 5.1 and the usual interpolation inequality for Sobolev spaces, if $\varepsilon>0$ is given, we have 


$$
\begin{aligned}
\left\|W^{I} \alpha\right\|_{\delta(r-|I|)} & =\left\|\Lambda^{\delta(r-|I|-1)} W^{I} \alpha\right\|_{\delta} \\
& \leq\left\|W^{I} \Lambda^{\delta(r-|I|-1)} \alpha\right\|_{\delta}+\left\|\left[\Lambda^{\delta(r-|I|-1)}, W^{I}\right] \alpha\right\|_{\delta} \\
& \leq\left\|W^{I} \Lambda^{\delta(r-|I|-1)} \alpha\right\|_{\delta}+\sum_{|J|<|I|}\left\|b_{J}(x, D) W^{J} \alpha\right\|_{\delta} \\
& \leq\left\|W^{I} \Lambda^{\delta(r-|I|-1)} \alpha\right\|_{\delta}+C \sum_{|J|<|I|}\left\|W^{J} \alpha\right\|_{\delta(r-|I|)} \\
& \leq\left\|W^{I} \Lambda^{\delta(r-|I|-1)} \alpha\right\|_{\delta}+C \varepsilon \sum_{|J|<|I|}\left\|W^{J} \alpha\right\|_{\delta(r-|J|)}+C_{\varepsilon} \sum_{|J|<|I|}\left\|W^{J} \alpha\right\|_{0} \\
& \leq\left\|W^{I} \Lambda^{\delta(r-|I|-1)} \alpha\right\|_{\delta}+C \varepsilon S_{|I|}+C_{\varepsilon} \sum_{|J|<|I|}\left\|W^{J} \alpha\right\|_{0} .
\end{aligned}
$$

By the (iterated) Poincaré inequality ([12] ), and by [29],

$$
C_{\varepsilon} \sum_{|J|<|I|}\left\|W^{J} \alpha\right\|_{0} \leq C_{\varepsilon} \sum_{|J|=r}\left\|W^{J} \alpha\right\|_{0} \leq C_{\varepsilon}\left(\langle\mathcal{L} \alpha, \alpha\rangle+\|\alpha\|_{0}^{2}\right)^{1 / 2},
$$

so that

$$
\left\|W^{I} \alpha\right\|_{\delta(r-|I|)} \leq\left\|W^{I} \Lambda^{\delta(r-|I|-1)} \alpha\right\|_{\delta}+C \varepsilon S_{|I|}+C_{\varepsilon}\left(\langle\mathcal{L} \alpha, \alpha\rangle+\|\alpha\|_{0}^{2}\right)^{1 / 2} .
$$

Let now $\eta \in \mathcal{D}(\mathbb{G})$ be such that $\eta \equiv 1$ on $\Omega$. We can write

$$
\begin{aligned}
& \left\|W^{I} \Lambda^{\delta(r-|I|-1)} \alpha\right\|_{\delta}=\left\|W^{I} \Lambda^{\delta(r-|I|-1)} \eta \alpha\right\|_{\delta} \\
& \quad \leq\left\|W^{I} \eta \Lambda^{\delta(r-|I|-1)} \alpha\right\|_{\delta}+\left\|W^{I}\left[\Lambda^{\delta(r-|I|-1)}, \eta\right] \alpha\right\|_{\delta} \\
& \quad \leq\left\|W^{I} \eta \Lambda^{\delta(r-|I|-1)} \alpha\right\|_{\delta}+\left\|\left[\Lambda^{\delta(r-|I|-1)}, \eta\right] W^{I} \alpha\right\|_{\delta}+\left\|\left[W^{I},\left[\Lambda^{\delta(r-|I|-1)}, \eta\right]\right] \alpha\right\|_{\delta} \\
& \quad=:\left\|W^{I} \eta \Lambda^{\delta(r-|I|-1)} \alpha\right\|_{\delta}+N_{1}+N_{2} .
\end{aligned}
$$

Keeping in mind that the operator $\left[\Lambda^{\delta(r-|I|-1)}, \eta\right]$ has order $\delta(r-|I|-1)-1$, again by interpolation and by the Poincaré inequality,

$$
\begin{aligned}
N_{1} & \leq\left\|W^{I} \alpha\right\|_{\delta(r-|I|)-1} \leq C \varepsilon\left\|W^{I} \alpha\right\|_{\delta(r-|I|)}+C_{\varepsilon}\left\|W^{I} \alpha\right\|_{0} \\
& \leq C \varepsilon S_{|I|}+C_{\varepsilon} \sum_{|J|=r}\left\|W^{J} \alpha\right\|_{0} \leq C \varepsilon S_{|I|}+C_{\varepsilon}\left(\langle\mathcal{L} \alpha, \alpha\rangle+\|\alpha\|_{0}^{2}\right)^{1 / 2}
\end{aligned}
$$

By Lemma 5.1 taking into account that $\left[\Lambda^{\delta(r-|I|-1)}, \eta\right]$ has order $\delta(r-|I|-1)-1$, there exist pseudodifferential operators $c_{J}(x, D)$ of order $\delta(r-|I|-1)-1$ such that

$$
\begin{aligned}
N_{2} & \leq \sum_{|J|<|I|}\left\|c_{J}(x, D) W^{J} \alpha\right\|_{\delta} \leq C \sum_{|J|<|I|}\left\|W^{J} \alpha\right\|_{\delta(r-|I|)-1} \\
& \leq C \sum_{|J|<|I|}\left\|W^{J} \alpha\right\|_{\delta(r-|J|)-1} \leq C \varepsilon \sum_{|J|<|I|}\left\|W^{J} \alpha\right\|_{\delta(r-|J|)}+C_{\varepsilon} \sum_{|J|<|I|}\left\|W^{J} \alpha\right\|_{0} \\
& \leq C \varepsilon S_{|I|}+C_{\varepsilon}\left(\langle\mathcal{L} \alpha, \alpha\rangle+\|\alpha\|_{0}^{2}\right)^{1 / 2},
\end{aligned}
$$


as above. Thus, combining [34], 35] and (36), we get

$\left\|W^{I} \Lambda^{\delta(r-|I|-1)} \alpha\right\|_{\delta} \leq\left\|W^{I} \eta \Lambda^{\delta(r-|I|-1)} \alpha\right\|_{\delta}+C \varepsilon S_{|I|}+C_{\varepsilon}\left(\langle\mathcal{L} \alpha, \alpha\rangle+\|\alpha\|_{0}^{2}\right)^{1 / 2}$.

Hence, by (33) and (37),

$$
\left\|W^{I} \alpha\right\|_{\delta(r-|I|)} \leq\left\|W^{I} \eta \Lambda^{\delta(r-|I|-1)} \alpha\right\|_{\delta}+C \varepsilon S_{|I|}+C_{\varepsilon}\left(\langle\mathcal{L} \alpha, \alpha\rangle+\|\alpha\|_{0}^{2}\right)^{1 / 2} .
$$

Remember now that, by a classical estimate ([10]), there exists $\delta \in(0,1)$ such that, if $\Omega_{0}$ is a bounded open set (which we choose such that supp $\eta \subset \Omega_{0}$ ), and $u \in \mathcal{D}\left(\Omega_{0}\right)$, then

$$
\|u\|_{\delta} \leq C\left(\sum_{j=1}^{m}\left\|W_{j} u\right\|_{0}+\|u\|_{0}\right),
$$

where $C=C\left(\Omega_{0}\right)$ is independent of $u \in \mathcal{D}\left(\Omega_{0}\right)$.

Going back to 377 and applying 39 to the components of $W^{I} \eta \Lambda^{\delta(r-|I|-1)} \alpha$ (which belongs to $\left.\mathcal{D}\left(\Omega_{0}\right)\right)$, we get

$$
\begin{aligned}
\left\|W^{I} \eta \Lambda^{\delta(r-|I|-1)} \alpha\right\|_{\delta} & \\
\leq & \sum_{|J|=|I|+1}\left\|W^{J} \eta \Lambda^{\delta(r-|I|-1)} \alpha\right\|_{0}+\left\|W^{I} \eta \Lambda^{\delta(r-|I|-1)} \alpha\right\|_{0} \\
\leq & \sum_{|J|=|I|+1}\left\|\eta \Lambda^{\delta(r-|I|-1)} W^{J} \alpha\right\|_{0}+\sum_{|J|=|I|+1}\left\|\left[W^{J}, \eta \Lambda^{\delta(r-|I|-1)}\right] \alpha\right\|_{0} \\
& \quad+\left\|W^{I} \eta \Lambda^{\delta(r-|I|-1)} \alpha\right\|_{0} \\
\leq & C \sum_{|J|=|I|+1}\left\|W^{J} \alpha\right\|_{\delta(r-|J|)}+\sum_{|J|=|I|+1}\left\|\left[W^{J}, \eta \Lambda^{\delta(r-|I|-1)}\right] \alpha\right\|_{0} \\
& +\left\|W^{I} \eta \Lambda^{\delta(r-|I|-1)} \alpha\right\|_{0} \\
= & C \sum_{|J|=|I|+1}\left\|W^{J} \alpha\right\|_{\delta(r-|J|)}+\sum_{|J|=|I|+1} N_{3, J}+N_{4} .
\end{aligned}
$$

By Lemma 5.1. keeping in mind that the operator $\eta \Lambda^{\delta(r-|I|-1)}$ has order $\delta(r-|I|-1)$, there exist pseudodifferential operators $c_{L}(x, D)$ of the same order such that

$$
\begin{aligned}
N_{4} & \leq\left\|\eta \Lambda^{\delta(r-|I|-1)} W^{I} \alpha\right\|_{0}+\left\|\left[W^{I}, \eta \Lambda^{\delta(r-|I|-1)}\right] \alpha\right\|_{0} \\
& \leq\left\|\eta \Lambda^{\delta(r-|I|-1)} W^{I} \alpha\right\|_{0}+\sum_{|L|<|I|}\left\|c_{L}(x, D) W^{L} \alpha\right\|_{0} \\
& \leq\left\|\eta \Lambda^{\delta(r-|I|-1)} W^{I} \alpha\right\|_{0}+\sum_{|L|<|I|}\left\|W^{L} \alpha\right\|_{\delta(r-|I|-1)} \leq C \sum_{|L| \leq|I|}\left\|W^{L} \alpha\right\|_{\delta(r-|L|-1)} \\
& \leq C \varepsilon \sum_{|L| \leq|I|}\left\|W^{L} \alpha\right\|_{\delta(r-|L|)}+C_{\varepsilon} \sum_{|L| \leq|I|}\left\|W^{L} \alpha\right\|_{0} \\
& \leq C \varepsilon S_{|I|}+C_{\varepsilon}\left(\langle\mathcal{L} \alpha, \alpha\rangle+\|\alpha\|_{0}^{2}\right)^{1 / 2}
\end{aligned}
$$


as above. Analogously, to estimate $N_{3, J}$, by Lemma 5.1, there exist pseudodifferential operators $d_{L}(x, D)$ of order $\delta(r-|I|-1)$ such that

$$
\begin{aligned}
N_{3, J} & \leq \sum_{|L| \leq|I|}\left\|d_{L}(x, D) W^{L} \alpha\right\|_{0} \leq C \sum_{|L| \leq|I|}\left\|W^{L} \alpha\right\|_{\delta(r-|I|-1)} \\
& \leq C \sum_{|L| \leq|I|}\left\|W^{L} \alpha\right\|_{\delta(r-|L|-1)} \leq C \varepsilon \sum_{|L| \leq|I|}\left\|W^{L} \alpha\right\|_{\delta(r-|L|)}+C_{\varepsilon} \sum_{|L| \leq|I|}\left\|W^{L} \alpha\right\|_{0} \\
& \leq C \varepsilon S_{|I|}+C_{\varepsilon}\left(\langle\mathcal{L} \alpha, \alpha\rangle+\|\alpha\|_{0}^{2}\right)^{1 / 2}
\end{aligned}
$$

as above. Combining (38), (40), 441) and (42) we get eventually

$$
\begin{aligned}
\left\|W^{I} \alpha\right\|_{\delta(r-|I|)} & \leq C \varepsilon S_{|I|}+C_{\varepsilon}\left(\|\mathcal{L} \alpha\|_{0}+\|\alpha\|_{0}\right) \\
& \leq C \varepsilon S_{r-1}+C_{\varepsilon}\left(\langle\mathcal{L} \alpha, \alpha\rangle+\|\alpha\|_{0}^{2}\right)^{1 / 2} .
\end{aligned}
$$

Summing up for $0 \leq|I| \leq r-1$ we obtain

$$
S_{r-1} \leq C \varepsilon S_{r-1}+C_{\varepsilon}\left(\langle\mathcal{L} \alpha, \alpha\rangle+\|\alpha\|_{0}^{2}\right)^{1 / 2},
$$

and hence (31) for a suitable choice of $\varepsilon>0$.

Step 2. If $\alpha \in \mathcal{D}^{\prime}\left(\Omega, \mathbb{R}^{N}\right)$, there exists $t \in \mathbb{R}$ such that

$$
W^{I} \alpha \in W_{\mathrm{loc}}^{t+\delta(r-|I|), 2}(\Omega) \quad \text { for }|I| \leq r .
$$

In fact, $\alpha \in W_{\text {loc }}^{-N, 2}(\Omega)$ for a suitable $N \geq 0$. Set now $t:=-N-r$. Since $W^{I} \alpha \in$ $W_{\text {loc }}^{-N-|I|, 2}(\Omega)$, the assertion follows since $W_{\text {loc }}^{-N-|I|, 2}(\Omega) \subset W_{\text {loc }}^{t+\delta(r-|I|), 2}(\Omega)$. Indeed, $-N-|I|-t-\delta(r-|I|)=r-|I|-\delta(r-|I|)=(r-|I|)(1-\delta) \geq 0$.

Suppose now $\mathcal{L} \alpha$ is smooth in $\Omega$. We want to show that

$$
W^{I} \alpha \in W_{\mathrm{loc}}^{t+\delta+\delta(r-|I|), 2}(\Omega) \text { for }|I| \leq r .
$$

We note first that, if $\psi \in \mathcal{D}(\Omega)$, then

$$
W^{I}(\psi \alpha) \in W^{t+\delta(r-|I|), 2}(\mathbb{G})
$$

for $|I| \leq r$. Indeed,

$$
W^{I}(\psi \alpha)=\sum_{\left|J_{1}\right|+\left|J_{2}\right| \leq|I|} c_{J_{1}, J_{2}} W^{J_{1}} \psi W^{J_{2}} \alpha \in W^{t+\delta(r-|I|), 2}(\mathbb{G}),
$$

since $W^{J_{2}} \alpha \in W_{\text {loc }}^{t+\delta\left(r-\left|J_{2}\right|\right), 2}(\Omega) \subset W_{\text {loc }}^{t+\delta(r-|I|), 2}(\Omega)$ for $\left|J_{2}\right| \leq|I|$. Let now $\eta, \psi \in$ $\mathcal{D}(\Omega)$ be such that $\eta \equiv 1$ on supp $\psi$. We have $\psi W^{I} \alpha=\psi W^{I}(\eta \alpha)$, so that 46) follows once we show that

$$
\psi W^{I}(\eta \alpha) \in W^{t+\delta+\delta(r-|I|), 2}(\mathbb{G}) \quad \text { for }|I| \leq r,
$$

i.e.

$$
\Lambda^{t+\delta+\delta(r-|I|)} \psi W^{I}(\eta \alpha) \in L^{2}(\mathbb{G}) \quad \text { for }|I| \leq r .
$$


Let now $I$ be fixed. For simplicity, we set $s:=t+\delta+\delta(r-|I|)$. We write

$$
\Lambda^{s} \psi W^{I}(\eta \alpha)=\psi \Lambda^{s} W^{I}(\eta \alpha)+\left[\Lambda^{s}, \psi\right] W^{I}(\eta \alpha) .
$$

By (47),

$$
\left[\Lambda^{s}, \psi\right] W^{I}(\eta \alpha) \in W^{t+\delta(r-|I|)-s+1,2}(\mathbb{G})=W^{1-\delta, 2}(\mathbb{G}) \subset L^{2}(\mathbb{G}) .
$$

Hence we are left with the proof that

$$
\psi \Lambda^{s} W^{I}(\eta \alpha) \in L^{2}(\mathbb{G}) .
$$

Let $\omega \in \mathcal{D}(\mathbb{G})$ be supported in the Euclidean unit ball at the origin, and assume $\int \omega(p) d p$ $=1$. If $\varepsilon>0$, we denote by $\omega_{\varepsilon}$ the Friedrichs mollifier $\omega_{\varepsilon}(p):=\varepsilon^{-n} \omega\left(\varepsilon^{-1} p\right)$. Denote by $*_{e}$ the convolution with respect to the usual Euclidean commutative group structure in $\mathbb{G} \equiv \mathbb{R}^{n}$. It is easy to see that the convolution operator $u \mapsto T_{\varepsilon} u:=u *_{e} \omega_{\varepsilon}$ is a pseudodifferential operator $T_{\varepsilon}(D) u$ with $T_{\varepsilon}(\xi)=\hat{\omega}(\varepsilon \xi) \in \mathcal{S}^{0}$. Moreover, $\left\{T_{\varepsilon}(\xi): \varepsilon \in\right.$ $(0,1)$ \} is bounded in $\mathcal{S}^{0}$.

More generally, we set $T_{\varepsilon}\left(\alpha_{1}, \ldots, \alpha_{N}\right):=\left(T_{\varepsilon} \alpha_{1}, \ldots, T_{\varepsilon} \alpha_{N}\right)$.

By a standard argument, (50) follows by showing that

$$
\left\|T_{\varepsilon} \psi \Lambda^{s} W^{I}(\eta \alpha)\right\|_{0} \leq C \quad \text { for } \varepsilon \in(0,1)
$$

Now

By Lemma 5.1

$$
T_{\varepsilon} \psi \Lambda^{s} W^{I}(\eta \alpha)=W^{I} T_{\varepsilon} \psi \Lambda^{s}(\eta \alpha)+\left[T_{\varepsilon} \psi \Lambda^{s}, W^{I}\right](\eta \alpha) .
$$

$$
\left[T_{\varepsilon} \psi \Lambda^{s}, W^{I}\right](\eta \alpha)=\sum_{|J|<|I|} c_{J, \varepsilon}(x, D) W^{J}(\eta \alpha)
$$

Since $c_{J, \varepsilon}(x, \xi)$ are equibounded in $\mathcal{S}^{s}$, by (47), $c_{J, \varepsilon}(x, D) W^{J}(\eta \alpha)$ are equibounded in $W^{t+\delta(r-|J|)-s, 2}(\mathbb{G}) \subset L^{2}(\mathbb{G})$, since $t+\delta(r-|J|)-s=\delta(|I|-|J|-1) \geq 0$ for $|J|<|I|$. Hence, the claim is proved by proving the $L^{2}$-equiboundedness of $W^{I} T_{\varepsilon} \psi \Lambda^{s}(\eta \alpha)$. Take $\psi_{1} \in \mathcal{D}(\Omega)$ with $\psi_{1} \equiv 1$ on an $\varepsilon$-neighborhood of supp $\psi$. We have

$$
\begin{aligned}
\left\|W^{I} T_{\varepsilon} \psi \Lambda^{s}(\eta \alpha)\right\|_{0}=\| & \Lambda^{\delta(r-|I|)} \Lambda^{-\delta(r-|I|)} \psi_{1} W^{I} T_{\varepsilon} \psi \Lambda^{s}(\eta \alpha) \|_{0} \\
\leq & \left\|\Lambda^{\delta(r-|I|)} \psi_{1} \Lambda^{-\delta(r-|I|)} W^{I} T_{\varepsilon} \psi \Lambda^{s}(\eta \alpha)\right\|_{0} \\
& +\left\|\Lambda^{\delta(r-|I|)}\left[\Lambda^{-\delta(r-|I|)}, \psi_{1}\right] W^{I} T_{\varepsilon} \psi \Lambda^{s}(\eta \alpha)\right\|_{0} \\
\leq & \left\|\Lambda^{\delta(r-|I|)} W^{I} \psi_{1} \Lambda^{-\delta(r-|I|)} T_{\varepsilon} \psi \Lambda^{s}(\eta \alpha)\right\|_{0} \\
& +\left\|\Lambda^{\delta(r-|I|)}\left[\psi_{1} \Lambda^{-\delta(r-|I|)}, W^{I}\right] T_{\varepsilon} \psi \Lambda^{s}(\eta \alpha)\right\|_{0} \\
& +\left\|\Lambda^{\delta(r-|I|)}\left[\Lambda^{-\delta(r-|I|)}, \psi_{1}\right] W^{I} T_{\varepsilon} \psi \Lambda^{s}(\eta \alpha)\right\|_{0} \\
\leq & \left\|\Lambda^{\delta(r-|I|)} W^{I} \psi_{1} \Lambda^{-\delta(r-|I|)} T_{\varepsilon} \psi \Lambda^{s}(\eta \alpha)\right\|_{0} \\
& +\left\|\Lambda^{\delta(r-|I|)}\left[\psi_{1} \Lambda^{-\delta(r-|I|)}, W^{I}\right] T_{\varepsilon} \psi \Lambda^{s}(\eta \alpha)\right\|_{0} \\
& +\left\|\Lambda^{\delta(r-|I|)}\left[\Lambda^{-\delta(r-|I|)}, \psi_{1}\right] T_{\varepsilon} \psi \Lambda^{s} W^{I}(\eta \alpha)\right\|_{0} \\
& +\left\|\Lambda^{\delta(r-|I|)}\left[\Lambda^{-\delta(r-|I|)}, \psi_{1}\right]\left[W^{I}, T_{\varepsilon} \psi \Lambda^{s}\right](\eta \alpha)\right\|_{0} \\
= & : S_{1}+S_{2}+S_{3}+S_{4} .
\end{aligned}
$$


Now

$$
S_{3} \leq C\left\|W^{I}(\eta \alpha)\right\|_{s-1}<\infty,
$$

by (47), since $s-1 \leq t+\delta(r-|I|)$. As for $S_{4}$, by Lemma 5.1, we can reduce ourselves to a sum of terms such as

$$
\left\|\Lambda^{\delta(r-|I|)}\left[\Lambda^{-\delta(r-|I|)}, \psi_{1}\right] c_{J, \varepsilon}(x, D) W^{J}(\eta \alpha)\right\|_{0}
$$

with $|J|<|I|$, where the symbols $c_{J, \varepsilon}(x, \xi)$ are equibounded in $\mathcal{S}^{s}$. Hence

$$
S_{4} \leq C \sum_{|J|<|I|}\left\|W^{J}(\eta \alpha)\right\|_{s-1}<\infty
$$

by (47), since $s-1 \leq t+\delta(r-|J|)$.

Moreover, by Lemma 5.1, $S_{2}$ can be estimated by a sum of $L^{2}$-norms of terms of the form

$$
\Lambda^{\delta(r-|I|)} c_{J}(x, D) W^{J} T_{\varepsilon} \psi \Lambda^{s}(\eta \alpha),
$$

where the $c_{J}(x, \xi)$ belong to $\mathcal{S}^{-\delta(r-|I|)}$ and $|J|<|I|$. In turn, by commutation and by Lemma 5.1, each of these terms can be estimated by the $L^{2}$-norm of

$$
\Lambda^{\delta(r-|I|)} c_{J}(x, D) T_{\varepsilon} \psi \Lambda^{s} W^{J}(\eta \alpha)
$$

plus a sum of $L^{2}$-norms of terms of the form

$$
\Lambda^{\delta(r-|I|)} c_{J}(x, D) b_{L, \varepsilon}(x, D) W^{L}(\eta \alpha),
$$

where $|L|<|J|$ and $b_{L, \varepsilon}(x, \xi)$ are equibounded in $\mathcal{S}^{s}$. All these terms can be estimated as $S_{3}$ and $S_{4}$. Indeed, for instance,

$$
\left\|\Lambda^{\delta(r-|I|)} c_{J}(x, D) T_{\varepsilon} \psi \Lambda^{s} W^{J}(\eta \alpha)\right\|_{0} \leq\left\|W^{J}(\eta \alpha)\right\|_{s}<\infty,
$$

since $s \leq t+\delta(r-|J|)$, for $t+\delta+\delta(r-|I|)-t-\delta(r-|J|)=\delta(|J|+1-|I|) \leq 0$.

Thus we are left with the estimate of

$$
S_{1}=\left\|W^{I} \psi_{1} \Lambda^{-\delta(r-|I|)} T_{\varepsilon} \psi \Lambda^{s}(\eta \alpha)\right\|_{\delta(r-|I|)} .
$$

We stress that $\psi_{1} \Lambda^{-\delta(r-|I|)} T_{\varepsilon} \psi \Lambda^{s}(\eta \alpha) \in \mathcal{D}\left(\mathbb{G}, \mathbb{R}^{N}\right)$, so that, by 31],

$$
\begin{aligned}
S_{1}^{2} \leq & \left\langle\mathcal{L} \psi_{1} \Lambda^{-\delta(r-|I|)} T_{\varepsilon} \psi \Lambda^{s}(\eta \alpha), \psi_{1} \Lambda^{-\delta(r-|I|)} T_{\varepsilon} \psi \Lambda^{s}(\eta \alpha)\right\rangle \\
& +\left\|\psi_{1} \Lambda^{-\delta(r-|I|)} T_{\varepsilon} \psi \Lambda^{s}(\eta \alpha)\right\|_{0}^{2}=: A_{1}+A_{2}
\end{aligned}
$$

Since the symbols of $\psi_{1} \Lambda^{-\delta(r-|I|)} T_{\varepsilon} \psi \Lambda^{s}$ are equibounded in $\mathcal{S}^{t+\delta}$, and $t+\delta \leq t+\delta r$,

$$
A_{2} \leq C\|\eta \alpha\|_{t+\delta r}^{2}<\infty .
$$

Let now $\eta_{1} \in \mathcal{D}(\Omega)$ be such that $\eta_{1} \equiv 1$ on supp $\eta$. We can write

$$
\begin{aligned}
\mathcal{L} \psi_{1} \Lambda^{-\delta(r-|I|)} T_{\varepsilon} \psi \Lambda^{s}(\eta \alpha)= & \psi_{1} \Lambda^{-\delta(r-|I|)} T_{\varepsilon} \psi \Lambda^{s} \eta \mathcal{L} \alpha \\
& +\left[\mathcal{L}, \psi_{1} \Lambda^{-\delta(r-|I|)} T_{\varepsilon} \psi \Lambda^{s} \eta\right] \eta_{1} \alpha=: B_{1}+B_{2}
\end{aligned}
$$


As above

$$
\left\langle B_{1}, \psi_{1} \Lambda^{-\delta(r-|I|)} T_{\varepsilon} \psi \Lambda^{s}(\eta \alpha)\right\rangle \leq C\|\eta \mathcal{L} \alpha\|_{t+\delta}\|\eta \alpha\|_{t+\delta r}<\infty .
$$

By Lemma 5.2, $\left\langle B_{2}, \psi_{1} \Lambda^{-\delta(r-|I|)} T_{\varepsilon} \psi \Lambda^{s}(\eta \alpha)\right\rangle$ can be written as a sum of terms of the form

$$
\left\langle b_{J, \varepsilon}(x, D) W^{J}\left(\eta_{1} \alpha\right) \mid c_{L}(x, D) W^{L} T_{\varepsilon} \psi \Lambda^{s}(\eta \alpha)\right\rangle,
$$

with $|J|<r,|L| \leq r$, the symbols $b_{J, \varepsilon}(x, \xi)$ and $c_{L}(x, \xi)$ being bounded in $\mathcal{S}^{t+\delta}$ and $\mathcal{S}^{-\delta(r-|I|)}$, respectively. As above

$$
\begin{aligned}
\left\langle B_{2}, \psi_{1} \Lambda^{-\delta(r-|I|)}\right. & \left.T_{\varepsilon} \psi \Lambda^{s}(\eta \alpha)\right\rangle \\
& \leq C \sum_{|J|<r,|L| \leq r}\left\|W^{J}\left(\eta_{1} \alpha\right)\right\|_{t+\delta}\left\|W^{L} T_{\varepsilon} \psi \Lambda^{s}(\eta \alpha)\right\|_{-\delta(r-|I|)} \\
& \leq C \sum_{|J|<r,|L| \leq r}\left\|W^{J}\left(\eta_{1} \alpha\right)\right\|_{t+\delta(r-|J|)}\left\|W^{L} T_{\varepsilon} \psi \Lambda^{s}(\eta \alpha)\right\|_{0} \\
& \leq \varepsilon \sum_{|L| \leq r}\left\|W^{L} T_{\varepsilon} \psi \Lambda^{s}(\eta \alpha)\right\|_{0}+C_{\varepsilon} \sum_{|J|<r}\left\|W^{J}\left(\eta_{1} \alpha\right)\right\|_{t+\delta(r-|J|)}
\end{aligned}
$$

Going back to 54, and taking into account the successive estimates, we get eventually

$$
\begin{aligned}
\left\|W^{I} T_{\varepsilon} \psi \Lambda^{s}(\eta \alpha)\right\|_{0} \leq & \varepsilon \sum_{|L| \leq r}\left\|W^{L} T_{\varepsilon} \psi \Lambda^{s}(\eta \alpha)\right\|_{0} \\
& +(\text { sum of equibounded terms })
\end{aligned}
$$

Summing up for $|I| \leq r$ and taking $\varepsilon$ suitably small, we completee the proof of [51, and hence that of (46). Now, by iteration, this shows that $\alpha$ is smooth and therefore that $\mathcal{L}$ is hypoelliptic.

Finally, let us prove that (i) implies (iv). To this end, we repeat the arguments of [1, Proposition 4.14]. For instance, if $a<Q$, by Theorem 3.1, for $\alpha \in \mathcal{D}\left(\mathbb{G}, \mathbb{R}^{N}\right)$ and $W^{I}$ again a homogeneous polynomial of degree $a$ in the horizontal derivatives, by (19), we can write

$$
W^{I} \alpha=W^{I} \mathcal{K} \mathcal{L} \alpha=\left(\sum_{j}(\mathcal{L} \alpha)_{j} * W^{I} K_{1 j}, \ldots, \sum_{j}(\mathcal{L} \alpha)_{j} * W^{I} K_{N j}\right)
$$

Since the $W^{I} K_{i j}$ 's are kernels of type 0 , the assertion follows by Proposition 1.9 of [6].

This completes the proof of the theorem.

\section{Appendix: $\Psi$ do's operators and commutation lemmata}

Following, e.g., [11, Ch. 18], if $s \in \mathbb{R}$, we denote by $\mathcal{S}^{s}$ the class of all symbols of order $s$, i.e. the class of all smooth functions $a=a(x, \xi)$ such that

$$
\left|\partial_{x}^{\beta} \partial_{\xi}^{\alpha} a(x, \xi)\right| \leq C_{\alpha, \beta}(1+|\xi|)^{s-|\alpha|}
$$


for all $x, \xi \in \mathbb{R}^{2 n+1}$ and for all nonnegative integral multi-indices $\alpha$ and $\beta$. The space $\mathcal{S}^{s}$ is a Fréchet space with respect to the seminorms

$$
\|a\|_{\alpha, \beta}=\sup _{x, \xi}\left|\partial_{x}^{\beta} \partial_{\xi}^{\alpha} a(x, \xi)\right|(1+|\xi|)^{|\alpha|-s} .
$$

As is customary, we denote by $a(x, D)$ the pseudodifferential operator associated with the symbol $a(x, \xi)$.

A pseudodifferential operator $P=P^{*}$ is said to be properly supported when for each compact $K$ there is a compact $K^{\prime}$ such that distributions supported in $K$ are mapped to distributions supported in $K^{\prime}$.

Lemma 5.1 ([15]). Let $I:=\left(i_{1}, \ldots, i_{h}\right)$ be a multi-index with $1 \leq i_{j} \leq 2 n$. Let $W^{I}:=$ $W_{i_{1}} \cdots W_{i_{h}}$ be a homogeneous polynomial in the horizontal vector fields $W_{1}, \ldots, W_{m}$, and let $\Lambda=\Lambda(x, D)$ be a properly supported pseudodifferential operator with a symbol $\Lambda(x, \xi) \in \mathcal{S}^{k}$. Then

$$
\left[W^{I}, \Lambda\right]=\sum_{|J| \leq|I|-1} b_{J}(x, D) W^{J},
$$

where the $b_{J}(x, D)$ 's are properly supported pseudodifferential operators with symbols $b_{J}(x, \xi) \in \mathcal{S}^{k}$. Moreover, if $\Lambda(x, \xi)$ lies in a bounded set of $\mathcal{S}^{k}$, then all the $b_{J}$ 's lie in a bounded set of $\mathcal{S}^{k}$.

Proof. We prove the assertion by induction on $h$. If $h=1$, then the statement follows since $\left[W_{j}, \Lambda\right]$ is a properly supported pseudodifferential operator of order $k$. Suppose (60) holds for $|I|=h$, and let us prove it for $|I|=h+1$. If $W^{I}:=W_{i_{1}} \cdots W_{i_{h}} W_{i_{h+1}}$, then (setting, for simplicity, $W_{i_{h+1}}:=W$ and $I_{0}:=\left(i_{1}, \ldots, i_{h}\right)$ )

$$
\begin{aligned}
{\left[W^{I}, \Lambda\right] } & =W^{I_{0}} W \Lambda-\Lambda W^{I_{0}} W=W^{I_{0}} \Lambda W+W^{I_{0}}[W, \Lambda]-\Lambda W^{I_{0}} W \\
& =\left[W^{I_{0}}, \Lambda\right] W+W^{I_{0}}[W, \Lambda]=\sum_{|J| \leq h-1} b_{J}(x, D) W^{J} W+W^{I_{0}} c(x, D) \\
& =\sum_{|J| \leq h-1} b_{J}(x, D) W^{J} W+c(x, D) W^{I_{0}}+\left[W^{I_{0}}, c(x, D)\right] \\
& =\sum_{|J| \leq h-1} b_{J}(x, D) W^{J} W+c(x, D) W^{I_{0}}+\sum_{|L| \leq h-1} c_{L}(x, D) W^{L},
\end{aligned}
$$

where the order of $b_{J}(x, D)$ is $k$, the order of $c(x, D)$ is $k$, and hence also the order of $c_{L}(x, D)$ is $k$. Finally, the last assertion follows again from the above identity if we keep in mind that the map from $\mathcal{S}^{s_{1}} \times \mathcal{S}^{s_{2}}$ to $\mathcal{S}^{s_{1}+s_{2}}$ associating with $a \in S^{s_{1}}$ and $b \in S^{s_{2}}$ the symbol of $a(x, D) \circ b(x, D)$ is continuous ([11, Theorem 18.1.8]).

Lemma 5.2. Let $I_{1}:=\left(i_{1}^{1}, \ldots, i_{h}^{1}\right)$ and $I_{2}:=\left(i_{1}^{2}, \ldots, i_{k}^{2}\right)$ be multi-indices with $1 \leq$ $i_{j}^{\ell} \leq 2 n$ and $\left|I_{1}\right|+\left|I_{2}\right| \leq 2 r$. Let $W^{I_{1}}:=W_{i_{1}^{1}} \cdots W_{i_{h}^{1}}$ and $W^{I_{2}}:=W_{i_{1}^{2}} \cdots W_{i_{k}^{2}}$ be homogeneous monomials in the horizontal vector fields $W_{1}, \ldots, W_{m}$, and let $\Lambda_{1}=\Lambda_{1}(x, D)$ and $\Lambda_{2}=\Lambda_{2}(x, D)$ be properly supported pseudodifferential operators with symbols 
$\Lambda_{1}(x, \xi) \in \mathcal{S}^{k}$ and $\Lambda_{2}(x, \xi) \in \mathcal{S}^{h}$, respectively. Let now $j_{0}$ and $\ell_{0}$ be non-negative integers such that $j_{0}+\ell_{0}=\left|I_{1}\right|+\left|I_{2}\right|-1$. Then we can write

$$
\begin{aligned}
\left\langle\left[W^{I_{1}}, \Lambda_{1}\right] u\right)\left|W^{I_{2}} \Lambda_{2} \phi\right\rangle_{\mathcal{D}^{\prime}(\mathbb{G}), \mathcal{D}(\mathbb{G})} & =\sum_{|J| \leq j_{0},|L| \leq \ell_{0}}\left\langle b_{J}(x, D) W^{J} u \mid c_{L}(x, D) W^{L} \phi\right\rangle_{\mathcal{D}^{\prime}(\mathbb{G}), \mathcal{D}(\mathbb{G})}
\end{aligned}
$$

for any $u \in \mathcal{E}^{\prime}(\mathbb{G})$ and $\phi \in \mathcal{D}(\mathbb{G})$, where the $b_{J}(x, D)$ 's and the $c_{L}(x, D)$ 's are properly supported pseudodifferential operators with symbols in $\mathcal{S}^{k}$ and $\mathcal{S}^{h}$, respectively. Moreover, if $\Lambda_{1}(x, \xi)$ and $\Lambda_{2}(x, \xi)$ lie respectively in bounded subsets of $\mathcal{S}^{k}$ and $\mathcal{S}^{h}$, then all the $b_{J}$ 's and the $c_{L}$ 's lie in bounded subsets of $\mathcal{S}^{k}$ and $\mathcal{S}^{h}$, respectively.

Proof. We argue by induction on $\left|I_{1}\right|+\left|I_{2}\right|$. If $\left|I_{1}\right|+\left|I_{2}\right|=1$, the assertion is straightforward, since the only non-trivial case we are dealing with is the scalar product $\left\langle\left[W_{j}, \Lambda_{1}\right] u, \Lambda_{2} u\right\rangle_{L^{2}(\mathbb{G})}$, which is already in the form of the right hand side of 61$]$. Suppose now (61) is true when $\left|I_{1}\right|+\left|I_{2}\right|=d<2 r$, and let us prove the assertion for $\left|I_{1}\right|+\left|I_{2}\right|=d+1$. Suppose also $j_{0}+\ell_{0}=d+1-1=d$. The following two cases can occur: either

$$
\left\langle\left[W W^{I_{1}^{*}}, \Lambda_{1}\right] u, W^{I_{2}} \Lambda_{2} u\right\rangle_{L^{2}(\mathbb{G})}
$$

or

$$
\left\langle\left[W^{I_{1}}, \Lambda_{1}\right] u, W W^{I_{2}^{*}} \Lambda_{2} u\right\rangle_{L^{2}(\mathbb{G})},
$$

where $W$ is one of the horizontal vector fields and $\left|I_{1}^{*}\right|+\left|I_{2}\right|=\left|I_{1}\right|+\left|I_{2}^{*}\right|=d$.

Consider first 63. By Lemma 5.1, there exist properly supported pseudodifferential operators $C_{J}(x, D)$ of order $k$ such that

$$
\begin{aligned}
\left\langle\left[W^{I_{1}}, \Lambda_{1}\right] u, W W^{I_{2}^{*}} \Lambda_{2} u\right\rangle_{L^{2}(\mathbb{G})}= & \sum_{|J|<\left|I_{1}\right|}\left\langle c_{J}(x, D) W^{J} u, W W^{I_{2}^{*}} \Lambda_{2} u\right\rangle_{L^{2}(\mathbb{G})} \\
= & \sum_{|J|<\left|I_{1}\right|}\left\langle W^{J} c_{J}(x, D) u, W W^{I_{2}^{*}} \Lambda_{2} u\right\rangle_{L^{2}(\mathbb{G})} \\
& +\sum_{|J|<\left|I_{1}\right|}\left\langle\left[c_{J}(x, D), W^{J}\right] u, W W^{I_{2}^{*}} \Lambda_{2} u\right\rangle_{L^{2}(\mathbb{G})} \\
= & : \sum_{|J|<\left|I_{1}\right|} S_{J}^{1}+\sum_{|J|<\left|I_{1}\right|} S_{J}^{2} .
\end{aligned}
$$

We notice that in $S_{J}^{2}$ we have $|J|+\left|I_{2}^{*}\right|+1 \leq\left|I_{1}\right|+\left|I_{2}^{*}\right|=d$, so that we can apply the inductive hypothesis replacing (for instance, if $j_{0}>0$ ) $j_{0}$ by $j_{0}-1$ and keeping $\ell_{0}$ unchanged. This is possible, since $j_{0}-1+\ell_{0}=d-1$. Hence $S_{J}^{2}$ is bounded by a sum of terms like

$$
\sum_{\left|L_{1}\right| \leq j_{0}-1,\left|L_{1}\right| \leq \ell_{0}}\left\langle b_{L_{1}}(x, D) W^{L_{1}} u, c_{L_{2}}(x, D) W^{L_{2}} u\right\rangle_{L^{2}(\mathbb{G})},
$$

which are among those on the right hand side of 61]. As for $S_{J}^{1}$, we notice that $|J|+$ $\left|I_{2}\right|+1 \leq\left|I_{1}\right|+\left|I_{2}\right|=d+1-1<2 r$. Thus, integrating by parts (to be formal: using iteratively the duality for distributional derivatives), we can write

$$
S_{J}^{1}= \pm\left\langle W^{K_{1}} c_{J}(x, D) u, W^{K_{2}} \Lambda_{2} u\right\rangle_{L^{2}(\mathbb{G})}
$$


with $\left|K_{1}\right| \leq j_{0}$ and $\left|K_{2}\right| \leq \ell_{0}$. We again apply Lemma 5.1 to both terms in the scalar product [65, and we get

$$
S_{J}^{1}=\sum_{\left|L_{1}\right| \leq\left|K_{1}\right|,\left|L_{2}\right| \leq\left|K_{2}\right|}\left\langle c_{L_{1}}(x, D) W^{L_{1}} u, c_{L_{2}}(x, D) W^{L_{2}} u\right\rangle_{L^{2}(\mathbb{G})},
$$

where the $c_{L_{1}}(x, D)$ 's and the $c_{L_{2}}(x, D)$ 's are properly supported pseudodifferential operators of order $k$. Again since $\left|L_{1}\right| \leq\left|K_{1}\right| \leq j_{0}$ and $\left|L_{2}\right| \leq\left|K_{2}\right| \leq \ell_{0}$, (66) is one of the terms on the right hand side of 61$]$.

Consider now the term 62. We can write

$$
\begin{aligned}
& \left\langle\left[W W^{I_{1}^{*}}, \Lambda_{1}\right] u, W^{I_{2}} \Lambda_{2} u\right\rangle_{L^{2}(\mathbb{G})} \\
& \quad=\left\langle W\left[W^{I_{1}^{*}}, \Lambda_{1}\right] u, W^{I_{2}} \Lambda_{2} u\right\rangle_{L^{2}(\mathbb{G})}+\left\langle\left[W, \Lambda_{1}\right] W^{I_{1} *} u, W^{I_{2}} \Lambda_{2} u\right\rangle_{L^{2}(\mathbb{G})} \\
& \quad=-\left\langle\left[W^{I_{1} *}, \Lambda_{1}\right] u, W W^{I_{2}} \Lambda_{2} u\right\rangle_{L^{2}(\mathbb{G})}+\left\langle\left[W, \Lambda_{1}\right] W^{I_{1} *} u, W^{I_{2}} \Lambda_{2} u\right\rangle_{L^{2}(\mathbb{G})} .
\end{aligned}
$$

The first term above has the form of 63 and can be handled precisely in the same way. The second term can be written as

$$
\begin{aligned}
& \left\langle\left[W, \Lambda_{1}\right] W^{I_{1}} u, W^{I_{2}} \Lambda_{2} u\right\rangle_{L^{2}(\mathbb{G})} \\
& \quad=\left\langle W^{I_{1}}\left[W, \Lambda_{1}\right] u, W^{I_{2}} \Lambda_{2} u\right\rangle_{L^{2}(\mathbb{G})}+\left\langle\left[\left[W, \Lambda_{1}\right], W^{I_{1}}\right] u, W^{I_{2}} \Lambda_{2} u\right\rangle_{L^{2}(\mathbb{G})} .
\end{aligned}
$$

But these terms have the form of those in $S_{J}^{1}$ and $S_{J}^{2}$ in 64), respectively, and can be written in the same way. This completes the proof of the lemma.

Acknowledgments. The authors are supported by MURST, Italy, by European project GALA, and by University of Bologna, Italy, funds for selected research topics.

The authors express their thanks to Ermanno Lanconelli, Jean Nourrigat and Fulvio Ricci for several stimulating discussions.

\section{References}

[1] Baldi, A., Franchi, B., Tesi, M. C.: Compensated compactness in the contact complex of Heisenberg groups. Indiana Univ. Math. J. 57, 133-185 (2008) Zbl 1143.43007 MR 2400254

[2] Baldi, A., Franchi, B., Tesi, M. C.: Fundamental solution and sharp $L^{p}$ estimates for Laplace operators in the contact complex of Heisenberg groups. Ricerche Mat. 55, 119-144 (2006) Zbl pre05177454 MR 2248167

[3] Bonfiglioli, A., Lanconelli, E., Uguzzoni, F.: Stratified Lie Groups and Potential Theory for Their Sub-Laplacians. Springer Monogr. Math., Springer, Berlin (2007) Zbl 1128.43001 MR 2363343

[4] Bourbaki, N.: Éléments de mathématique. XXVI. Groupes et algèbres de Lie. Chapitre 1: Algèbres de Lie. Actualités Sci. Ind. 1285, Hermann, Paris (1960) Zbl 0199.35203 MR 0132805

[5] Fefferman, C. L., Phong, D. H.: Subelliptic eigenvalue problems. In: Conference on Harmonic Analysis in Honor of Antoni Zygmund, Vol. I, II (Chicago, IL, 1981), Wadsworth Math. Ser., Wadsworth, Belmont, CA, 590-606 (1983) Zbl 0503.35071 MR 0730094 
[6] Folland, G. B.: Subelliptic estimates and function spaces on nilpotent Lie groups. Ark. Mat. 13, 161-207 (1975) Zbl 0312.35026 MR 0494315

[7] Folland, G. B., Stein, E. M.: Hardy Spaces on Homogeneous Groups. Math. Notes 28, Princeton Univ. Press, Princeton, NJ (1982) Zbl 0508.42025 MR 0657581

[8] Franchi, B., Serapioni, R., Serra Cassano, F.: On the structure of finite perimeter sets in step 2 Carnot groups. J. Geom. Anal. 13, 421-466 (2003) Zbl 1064.49033 MR 1984849

[9] Helffer, B., Nourrigat, J.: Hypoellipticité maximale pour des opérateurs polynômes de champs de vecteurs. Progr. Math. 58, Birkhäuser Boston, Boston, MA (1985) Zbl 0568.35003 MR 0897103

[10] Hörmander, L.: Hypoelliptic second order differential equations. Acta Math. 119, 147-171 (1967) Zbl 0156.10701 MR 0222474

[11] Hörmander, L.: The Analysis of Linear Partial Differential Operators. I. Grundlehren Math. Wiss. 256, Springer, Berlin (1990) Zbl 0712.35001 MR 1065993

[12] Jerison, D.: The Poincaré inequality for vector fields satisfying Hörmander's condition. Duke Math. J. 53, 503-523 (1986) Zbl 0614.35066 MR 0850547

[13] Kohn, J. J.: Pseudo-differential operators and hypoellipticity. In: Partial Differential Equations (Berkeley, CA, 1971), Proc. Sympos. Pure Math. 23, Amer. Math. Soc., Providence, RI, 61-69 (1973) Zbl 0262.35007 MR 0338592

[14] Luo, X.: Liouville's theorem for homogeneous differential operators. Comm. Partial Differential Equations 22, 1837-1848 (1997) Zbl 0908.35027 MR 1629494

[15] Nourrigat, J.: Subelliptic Estimates for Systems of Pseudo-Differential Operators. Notas de curso 20, Recife (1982)

[16] Ponge, R.: Heisenberg calculus and spectral theory of hypoelliptic operators on Heisenberg manifolds. Mem. Amer. Math. Soc. 194, no. 906 (2008) Zbl 1143.58014 MR 2417549

[17] Ricci, F.: Sub-Laplacians on Nilpotent Lie Groups (Appendix: Hörmander's Theorem). Lecture Notes at the Scuola Normale Superiore, Pisa (2002-2003); http://homepage.sns.it/fricci/corsi.html.

[18] Rothschild, L. P., Stein, E. M.: Hypoelliptic differential operators and nilpotent groups. Acta Math. 137, 247-320 (1976) Zbl 0346.35030 MR 0436223

[19] Rumin, M.: Formes différentielles sur les variétés de contact. J. Differential Geom. 39, 281330 (1994) Zbl 0973.53524 MR 1267892

[20] Rumin, M.: Around heat decay on forms and relations of nilpotent Lie groups. In: Séminaire de Théorie Spectrale et Géométrie, Vol. 19, Année 2000-2001, Univ. Grenoble I, 123-164 (2001) Zbl 1035.58021 MR 1909080

[21] Stein. E. M.: Harmonic Analysis: Real-Variable Methods, Orthogonality, and Oscillatory Integrals. Princeton Math. Ser. 43, Princeton Univ. Press, Princeton, NJ (1993) Zbl 0821.42001 MR 1232192 